\title{
SHORT-RUN CONTRACTS ON EUROPEAN UNION MARKET AND GOOD FAITH PRINCIPLE IN THE REALITY OF GEORGIAN ECONOMY
}

\author{
NINO MELITAURI \\ PhD student \\ Ivane Javakhishvili Tbilisi State University, Georgia \\ melitaurinina@gmail.com
}

Abstract. How are transactions conducted on Georgian consumption market and the European Union internal market? During exportation of some agro products from Georgia to the EU it was found out that relevant trade habits in making a deal varies greatly for the 'two Economies' and the difference is significant. Apparently, this makes an issue for present-day commerce and, presumably, the disparities, which have just been noted, risk to pose a serious threat to trade relationships between the two economic Units. Therefore, this Article aims at looking around the differences. I will attempt to explain the flexibility of the contemporary 'simplified-type transactions' conducted in the EU, characterized by disappearance of traditional formal stages of contracting (negotiations, contract-signing, etc.). In this Article, I will also show out that these relationship-building (stemming from the 'brand-new transactions') is more consecutive, logical and dynamic, leaving less room for the breach of a contract or principle of good faith.

KEYWORDS: DCFTA, PRINCIPLE OF GOOD FAITH, EU-GEORGIA TRANSNATIONAL COMMERCE, TRADE DEALS.

For citation: Melitauri N. (2020), Short-Run Contracts on European Union Market and Good Faith Principle in the Reality of Georgian Economy. Globalization and Business, №9, pp. 129-134 (In Georgian). https://doi.org/10.35945/gb.2020.09.016 


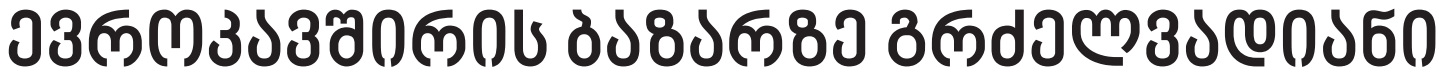

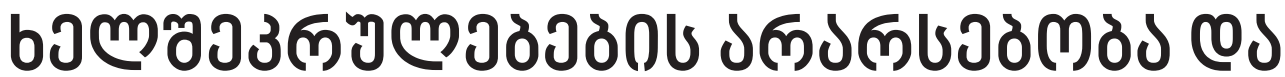

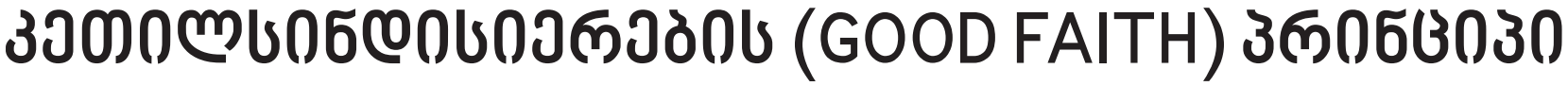

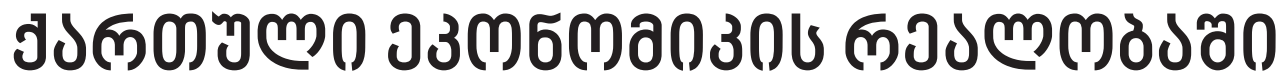

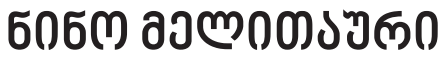

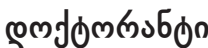

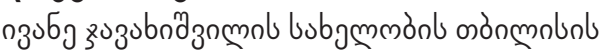

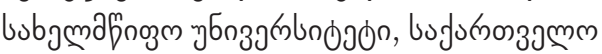

melitaurinina@gmail.com

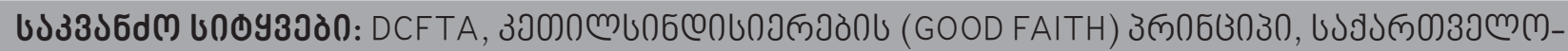

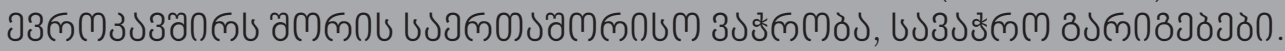

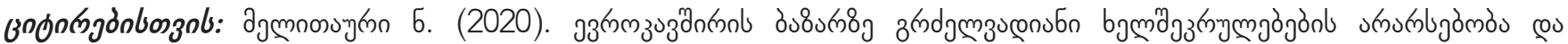

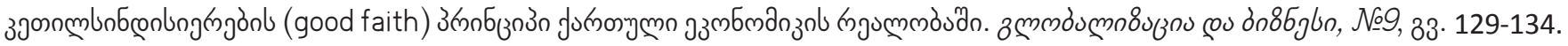
https://doi.org/10.35945/gb.2020.09.016

\section{əอง১3১ल00}

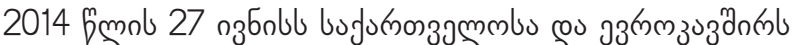

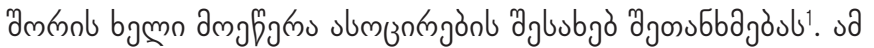

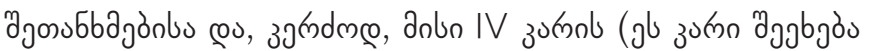

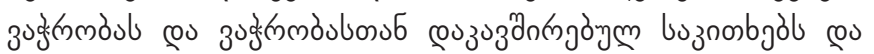

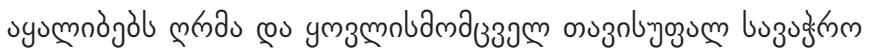
unzrrogl [Deep and Comprehensive Free Trade Area -

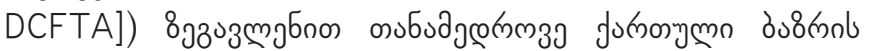
bo by

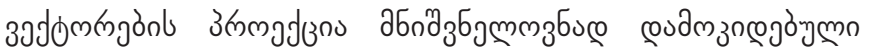

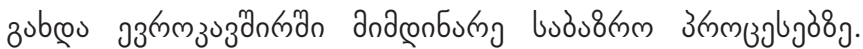

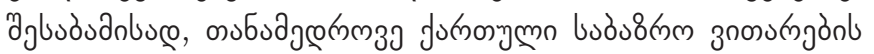

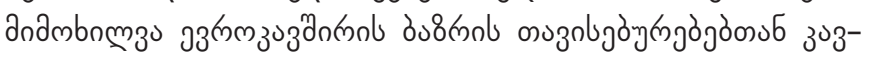

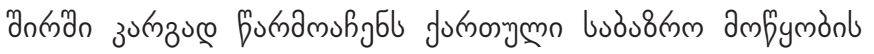

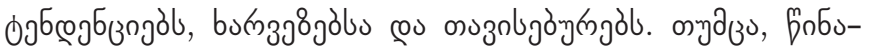

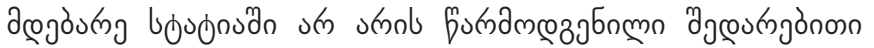

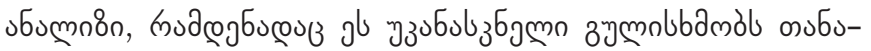

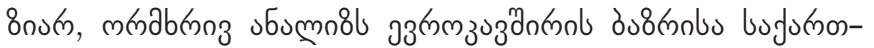

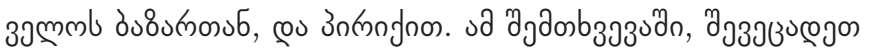

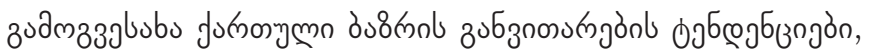

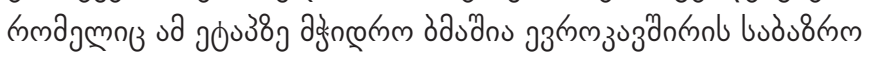

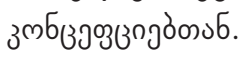

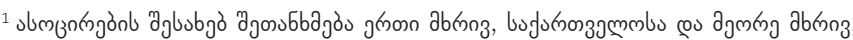

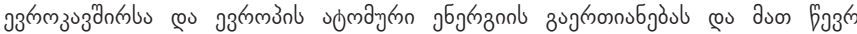

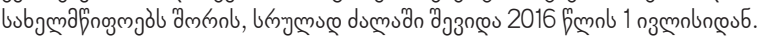

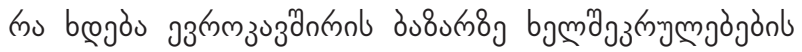

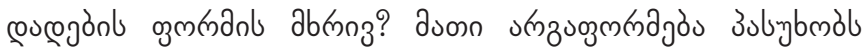

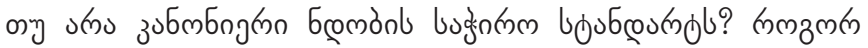

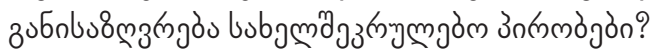

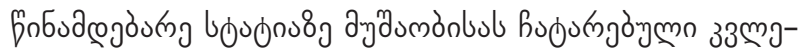

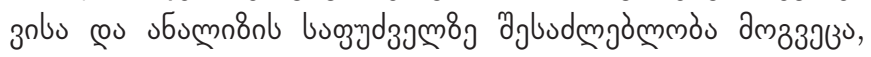

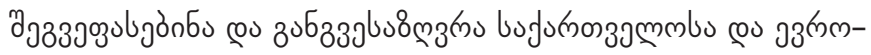

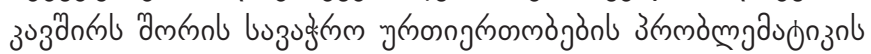

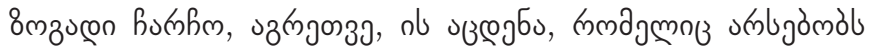

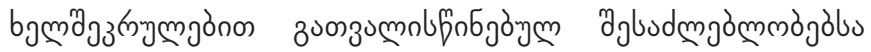

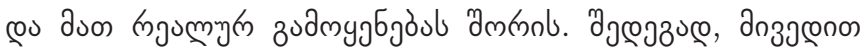

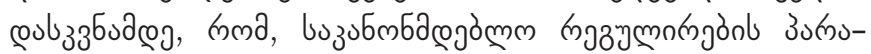

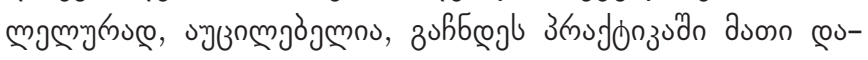

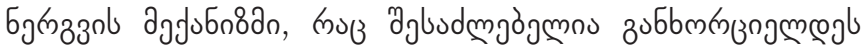
DCFTA-non zuonzu molbfóб.

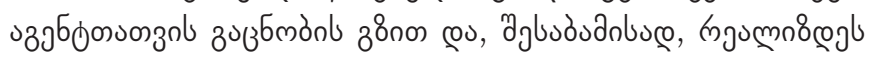

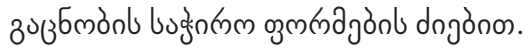

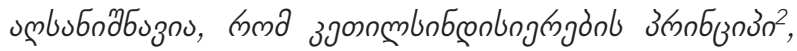

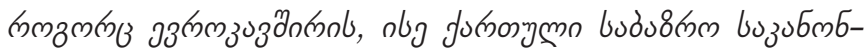

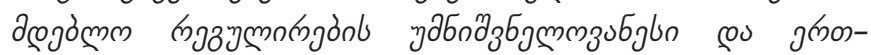

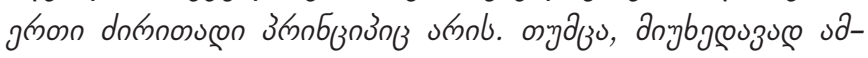

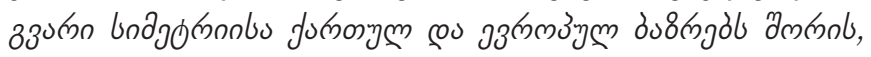

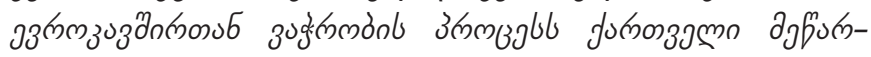

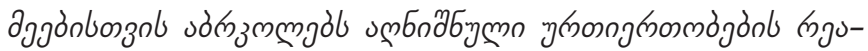

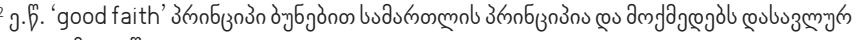

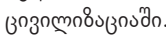




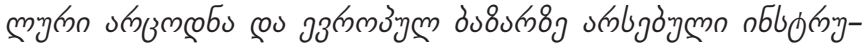

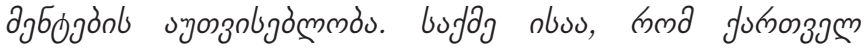

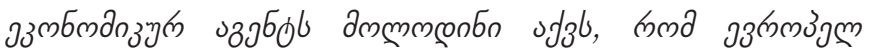

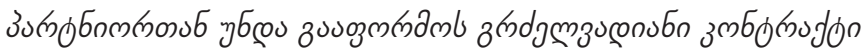

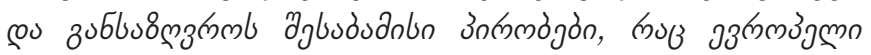

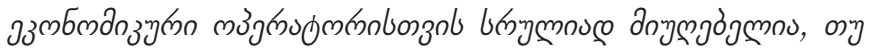

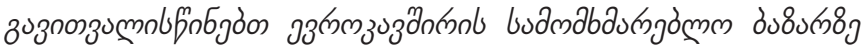

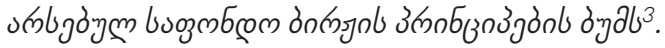

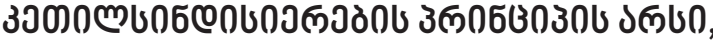

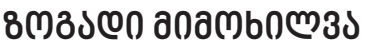

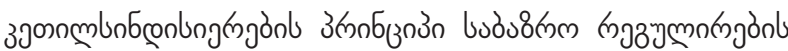

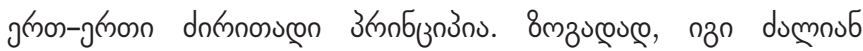

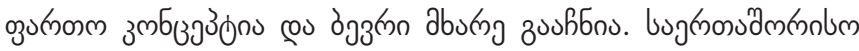

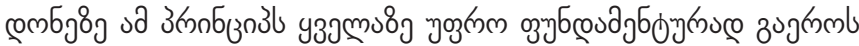

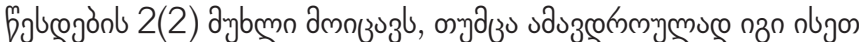

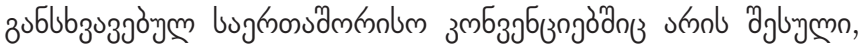

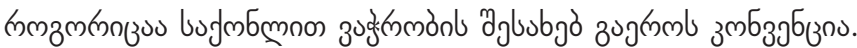

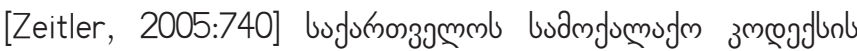

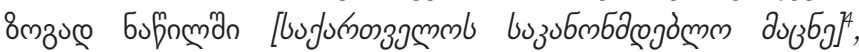

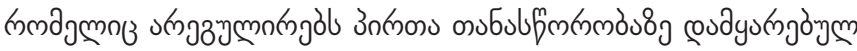
зg

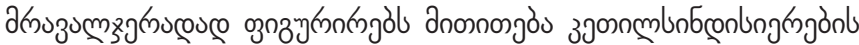

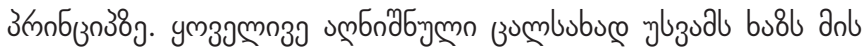

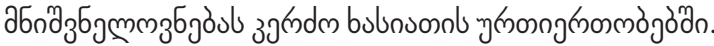

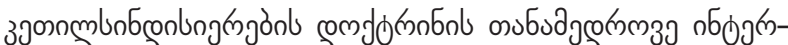

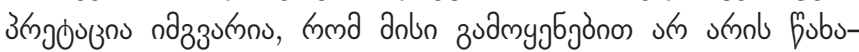

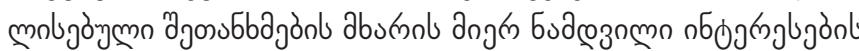

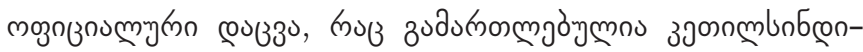

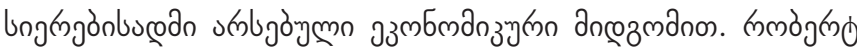

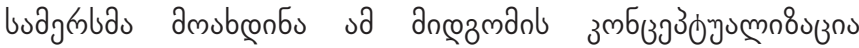

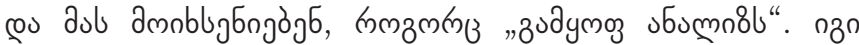

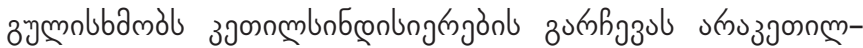

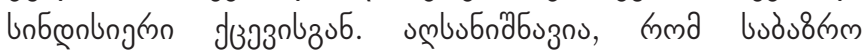

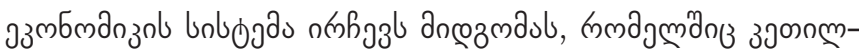

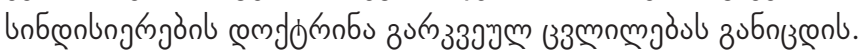

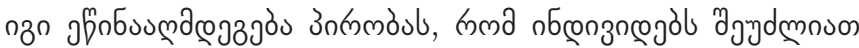

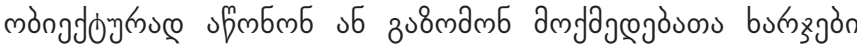

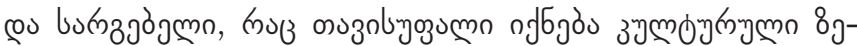

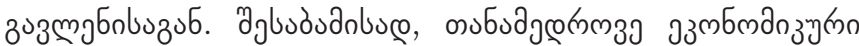

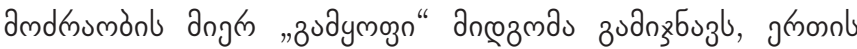

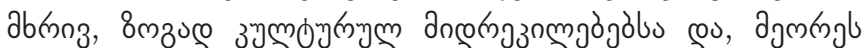

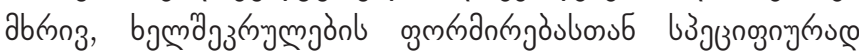

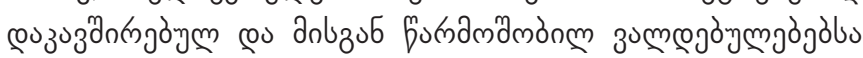

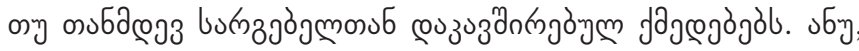

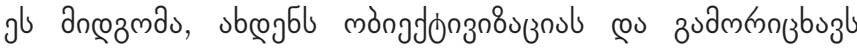

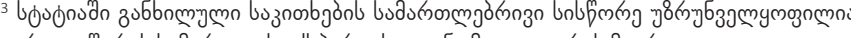

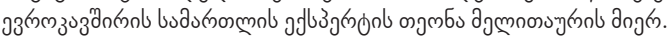

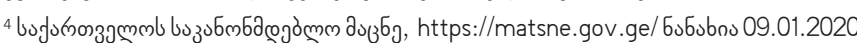

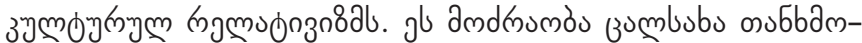

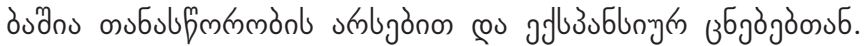
[Houh, 2003:1029].

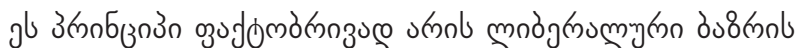

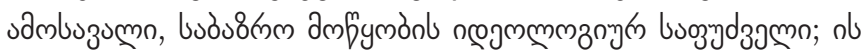

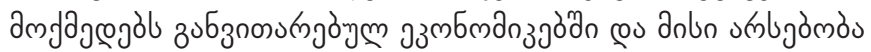

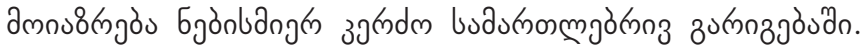

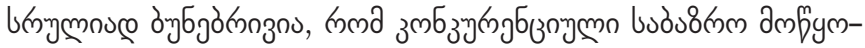

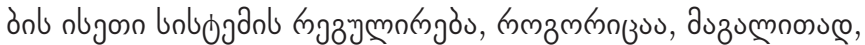

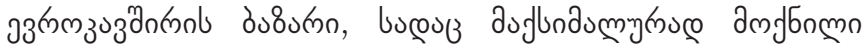

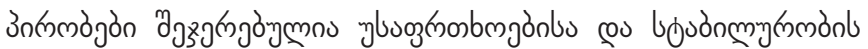

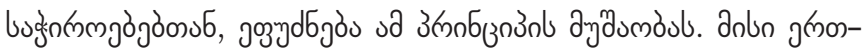

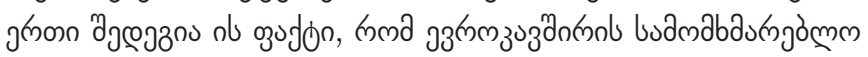
उu8s моुnznum

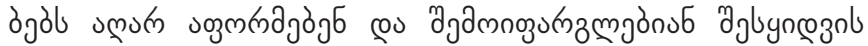

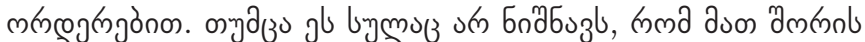

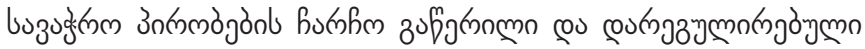

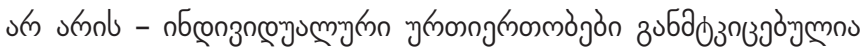

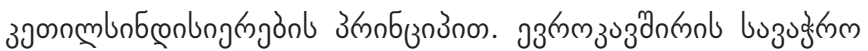

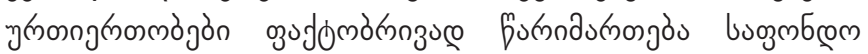

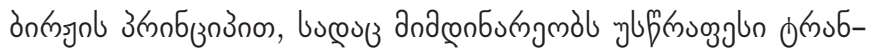

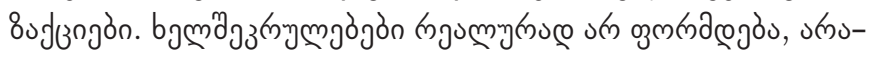

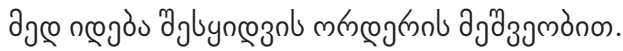

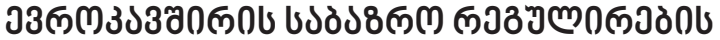

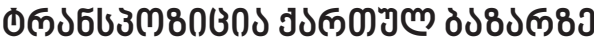

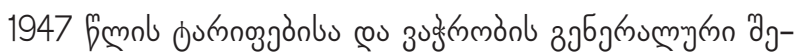

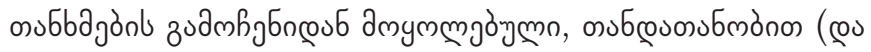

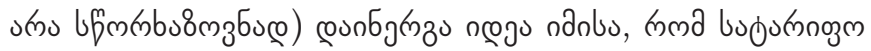

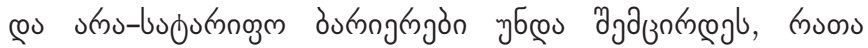

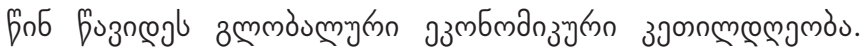
[Bhala,1997:190].

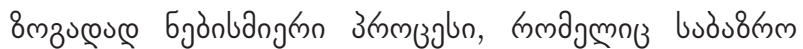

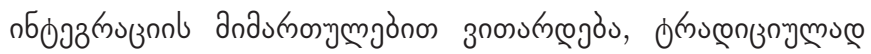

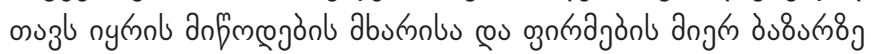

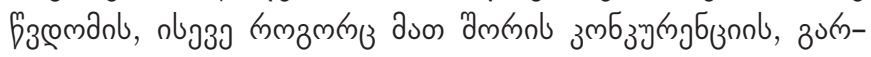
ygam. [Moloney, 2004:11].

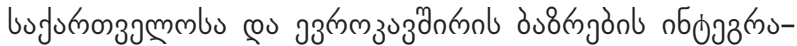

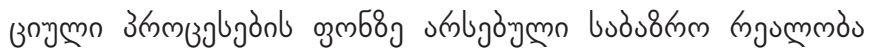

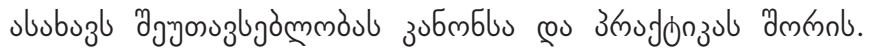

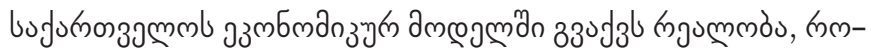

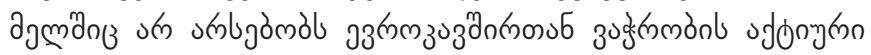

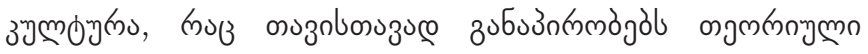

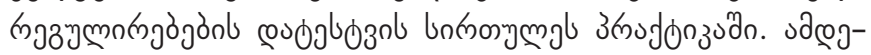

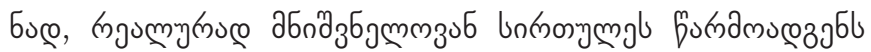

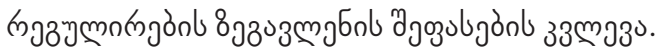

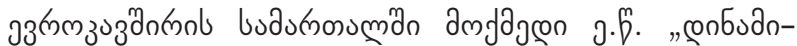

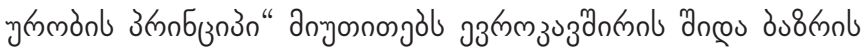




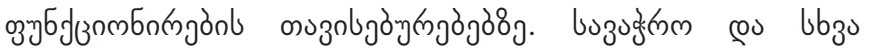
зg

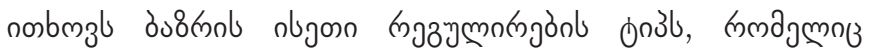

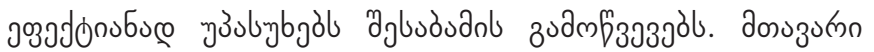
зuдm

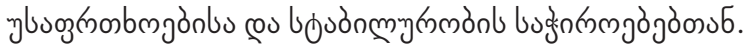

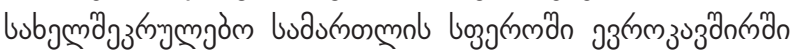

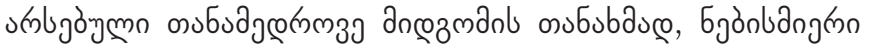

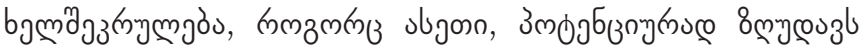

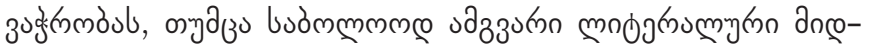

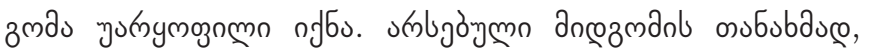

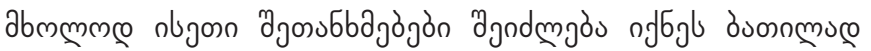

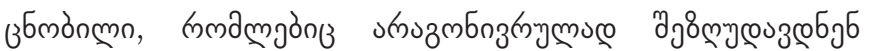

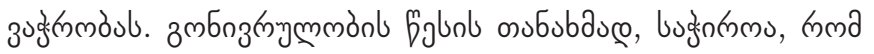

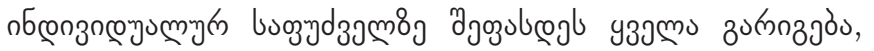

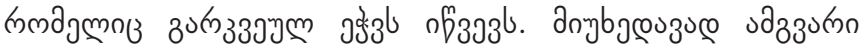

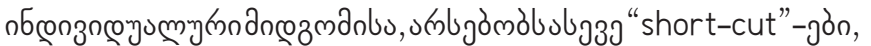
फलдm

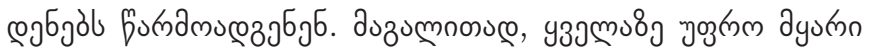

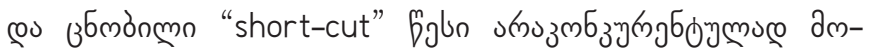

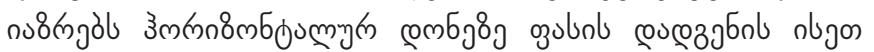

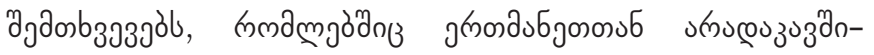

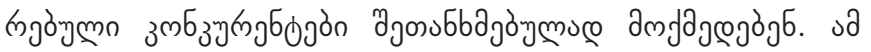

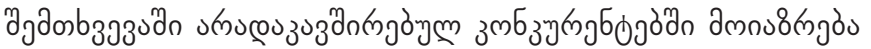

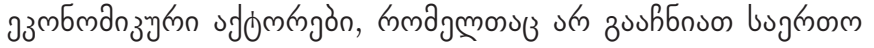

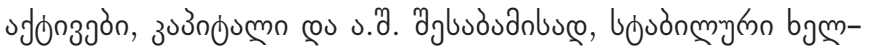

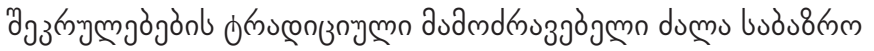

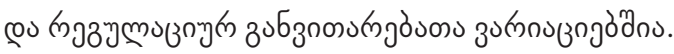

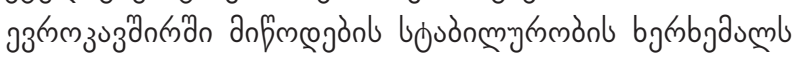
sonkm

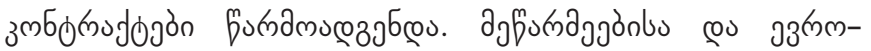

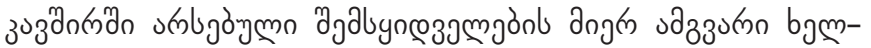

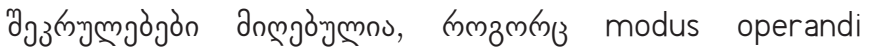

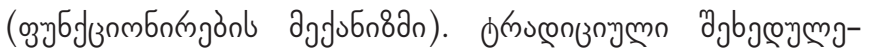

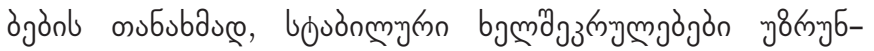

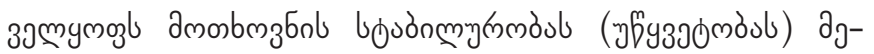

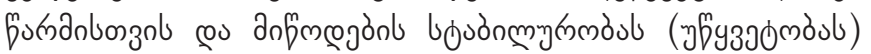
gyalugn@

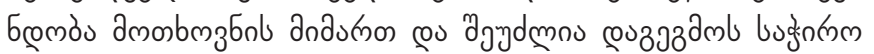

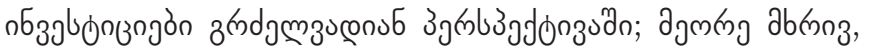

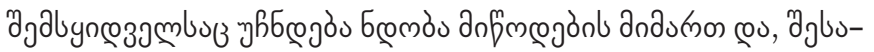

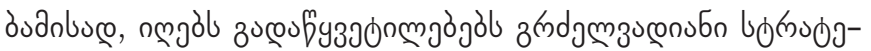

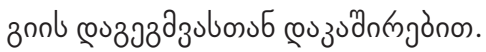

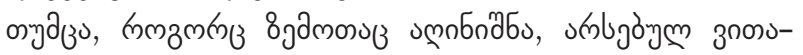

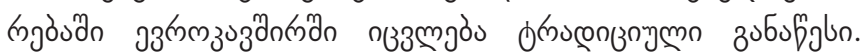

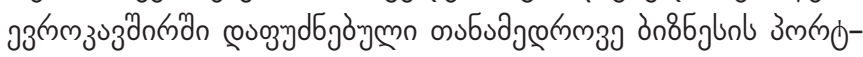

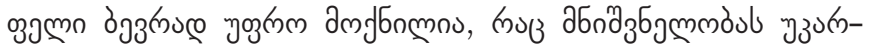

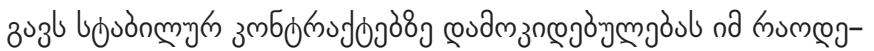

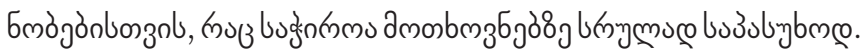

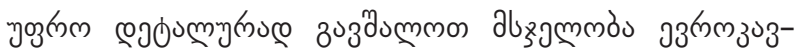

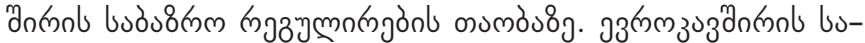

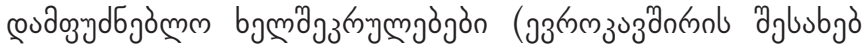

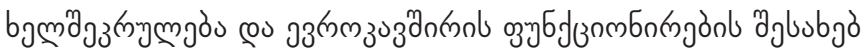

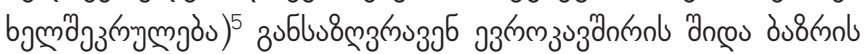

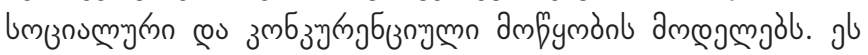

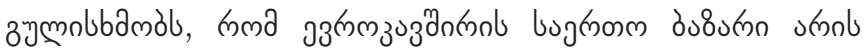

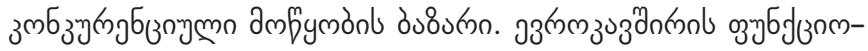

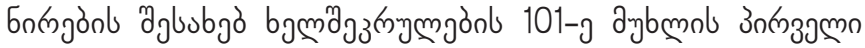

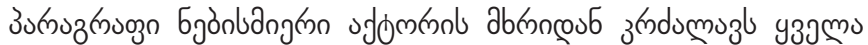

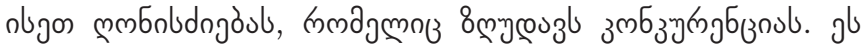

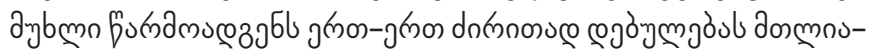

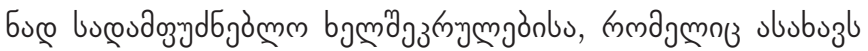

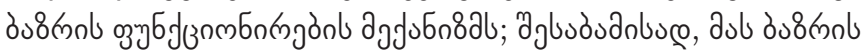

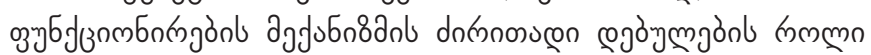

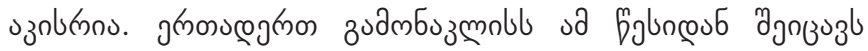

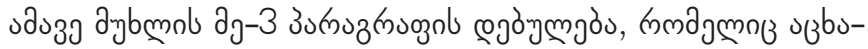

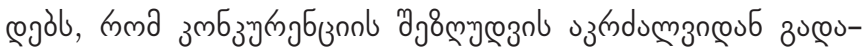

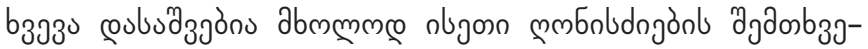

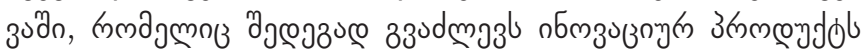

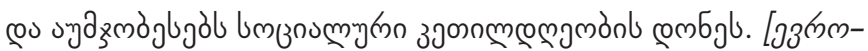

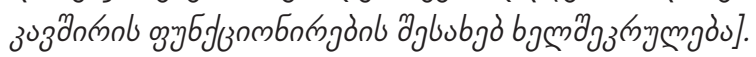

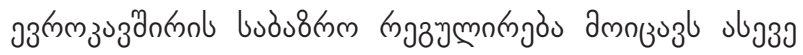

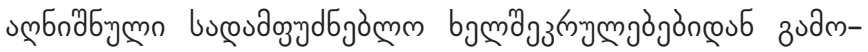

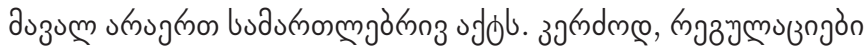

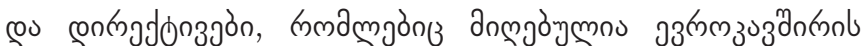

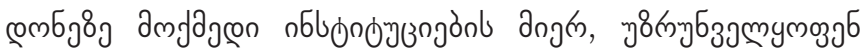

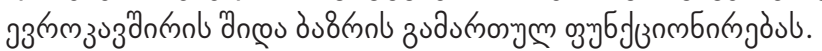

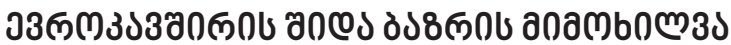

2.

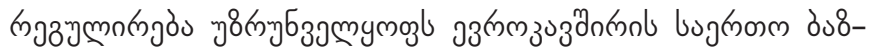

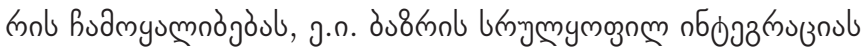

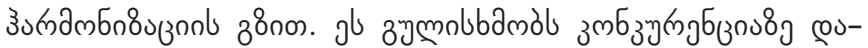

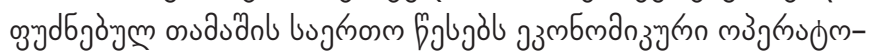
mgànbonzol.

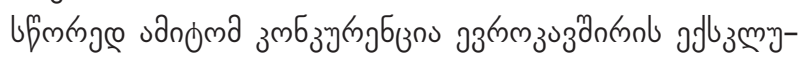

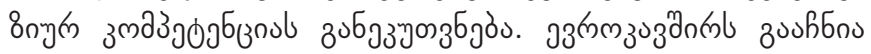

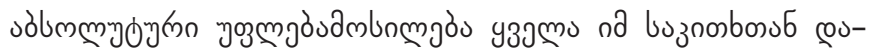

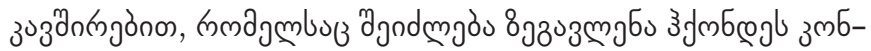

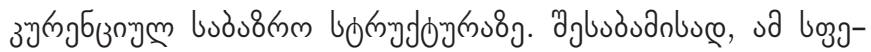

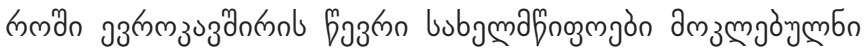

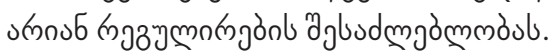

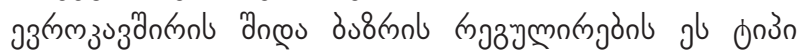

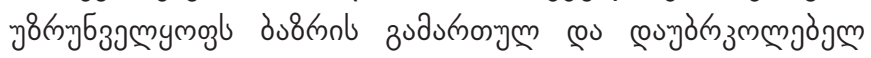

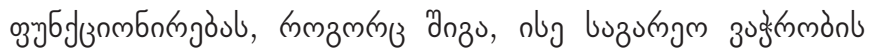

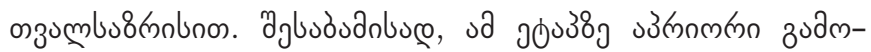

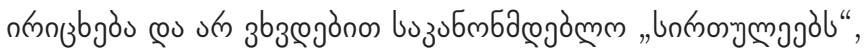

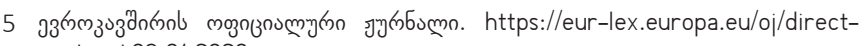
access.html 08.01.2020 


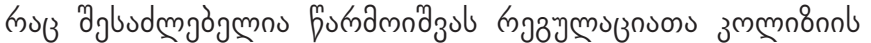

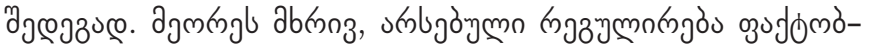

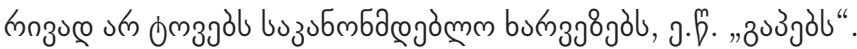

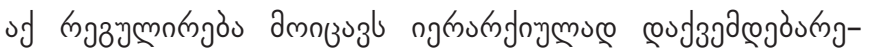

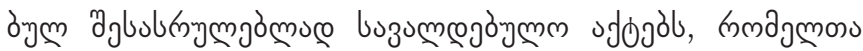

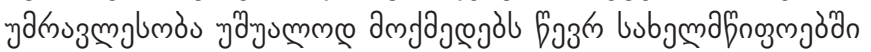

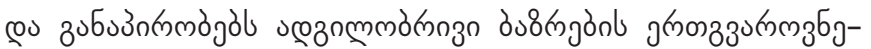

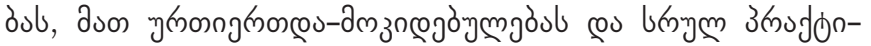

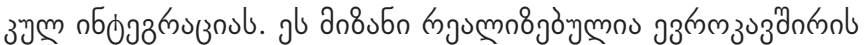

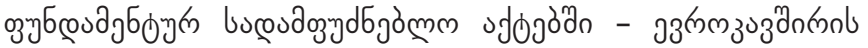
चjbubjo by

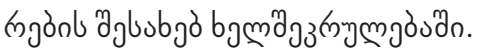

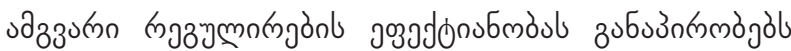

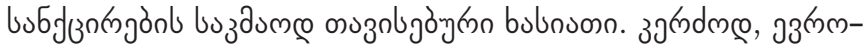

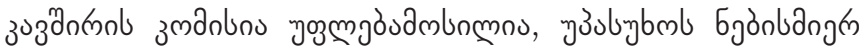

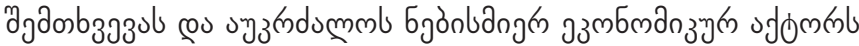

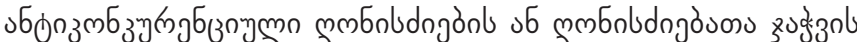
zubbmingrammgos.

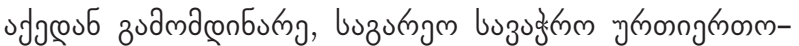

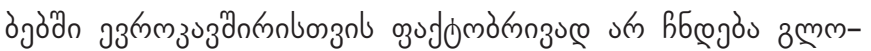

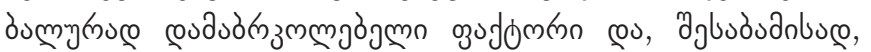

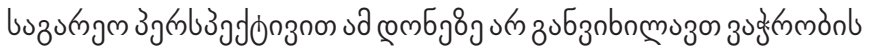

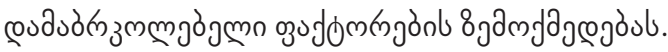

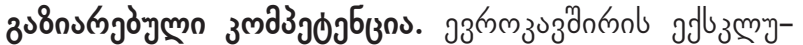

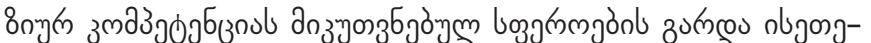

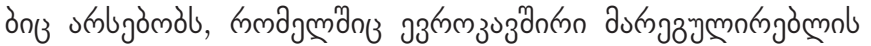

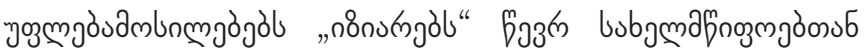

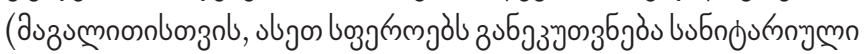

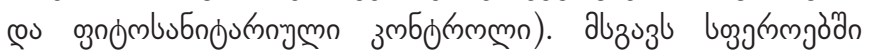

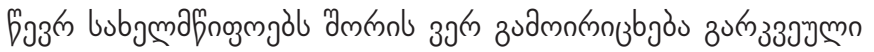

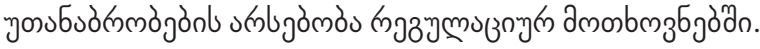

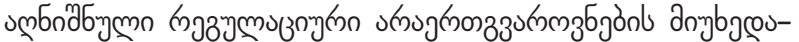

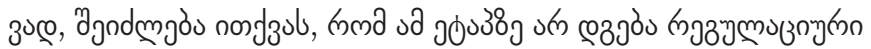

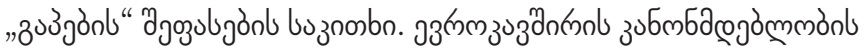

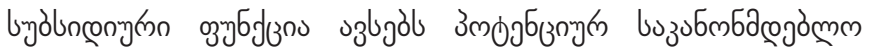

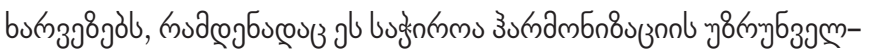

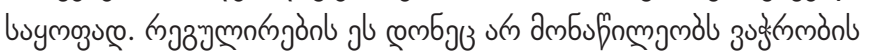

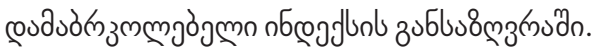

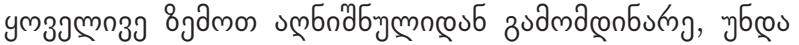

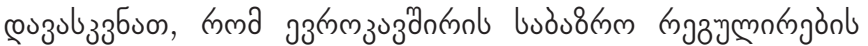

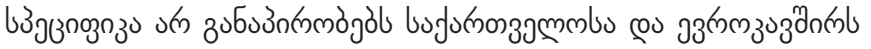

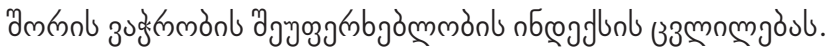

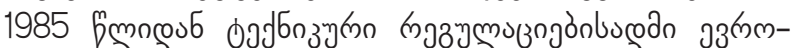

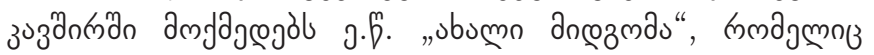

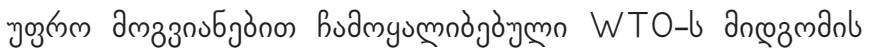

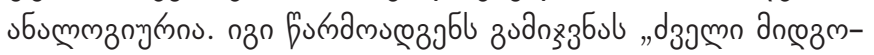

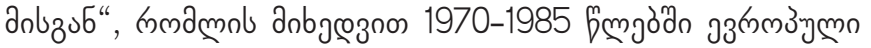

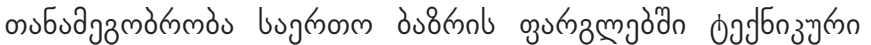

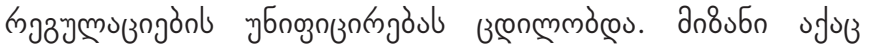

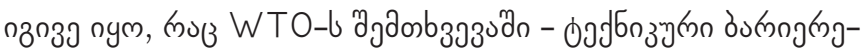

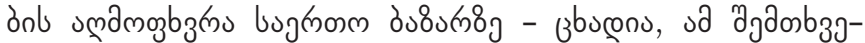

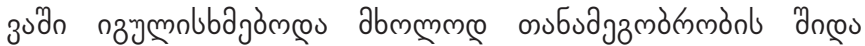

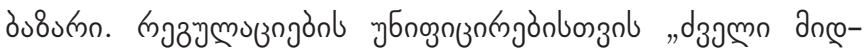

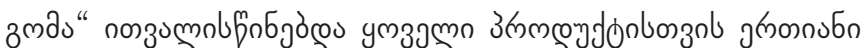

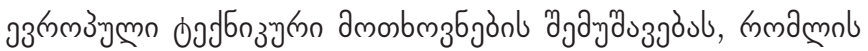

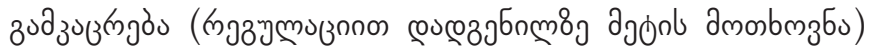

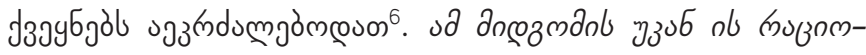

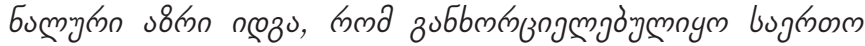

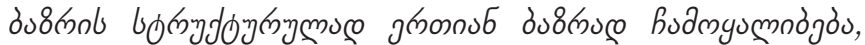

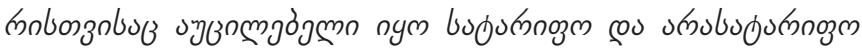

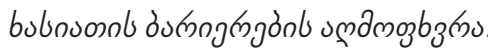

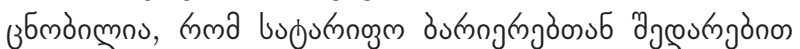

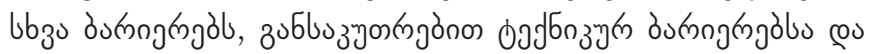

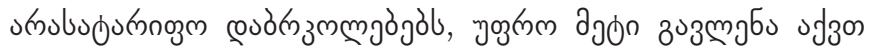

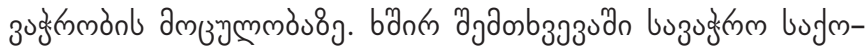

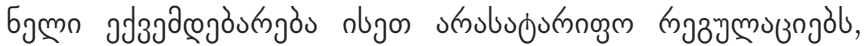

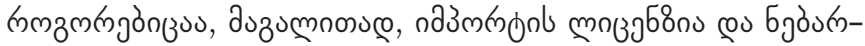

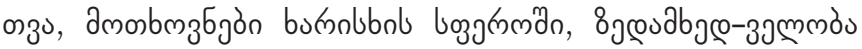

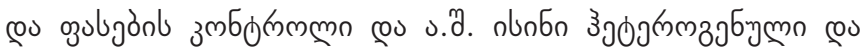

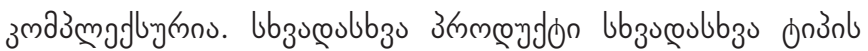

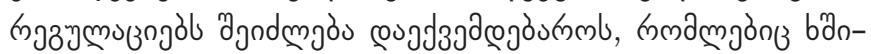

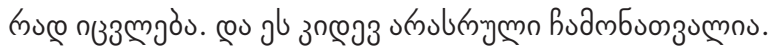

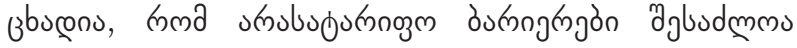

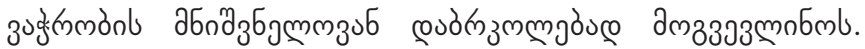

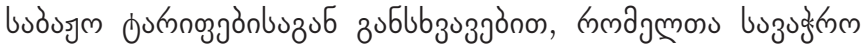

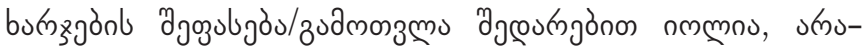

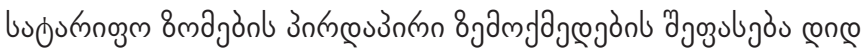

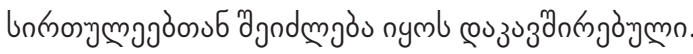

uмб

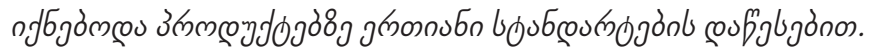

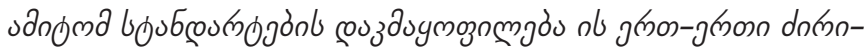

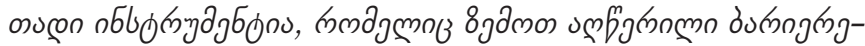

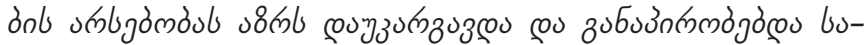

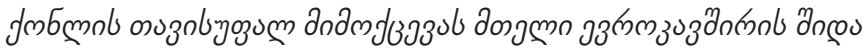
juornol aubagouonon.

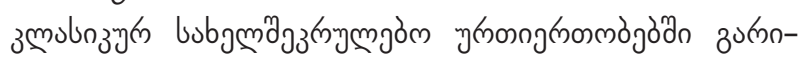

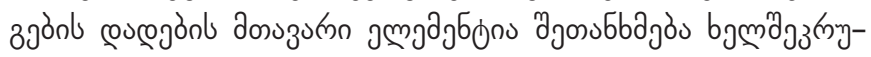

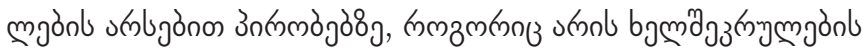

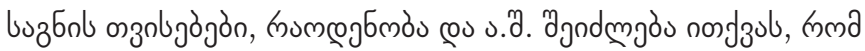

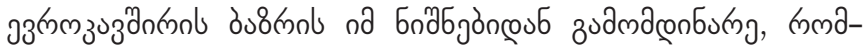

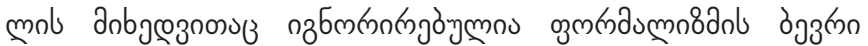

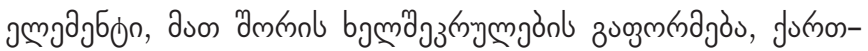

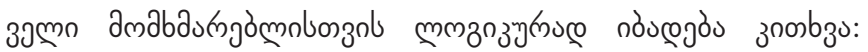

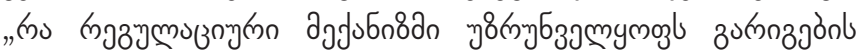

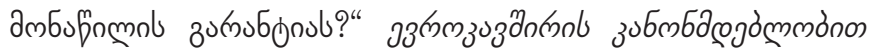

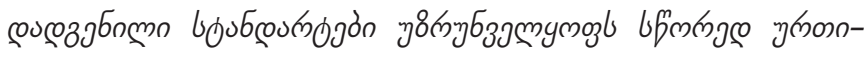

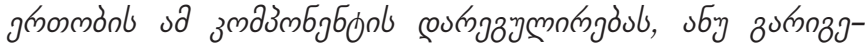

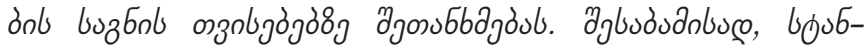

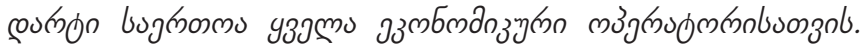

${ }^{6}$ http://asocireba.ge/files/file_2864583.pdf. бububno 06.10.2019. 


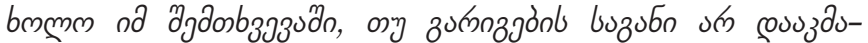

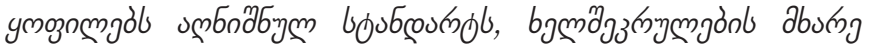

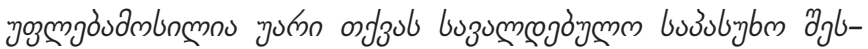

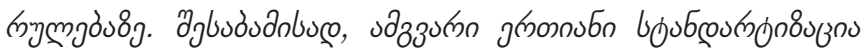

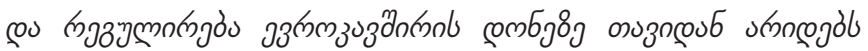

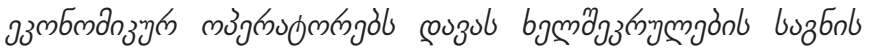

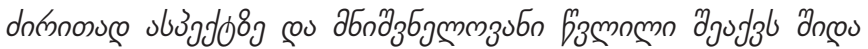

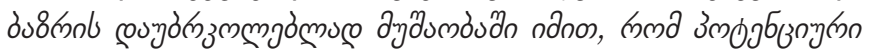

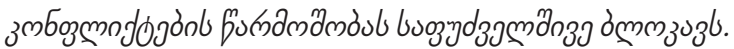

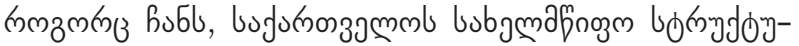

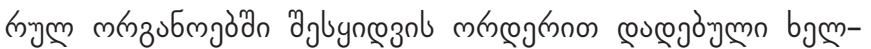

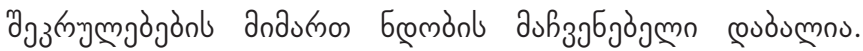

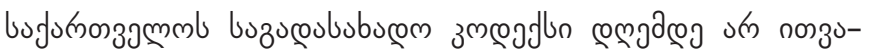

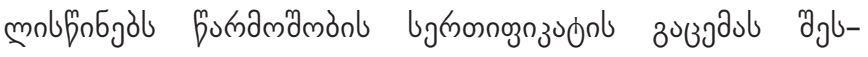

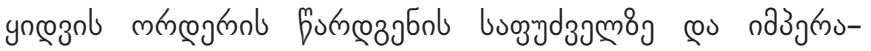

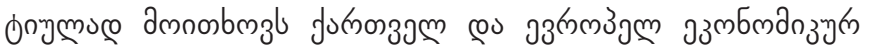

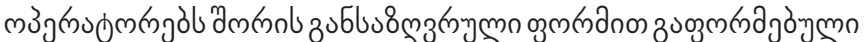

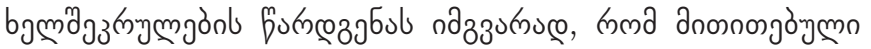

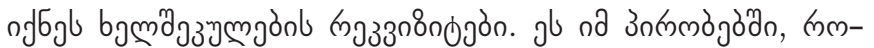

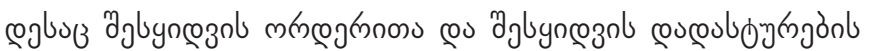

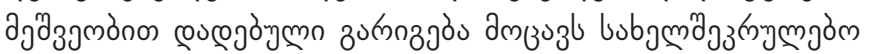

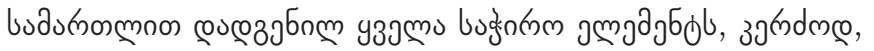

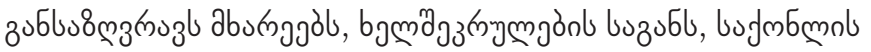

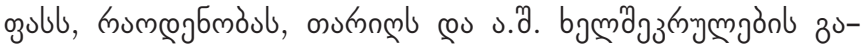

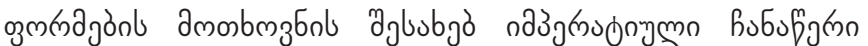

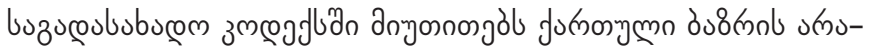

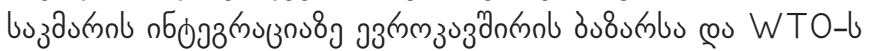

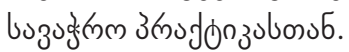

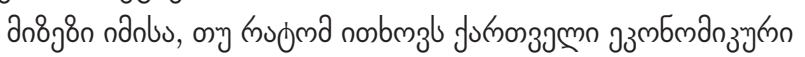

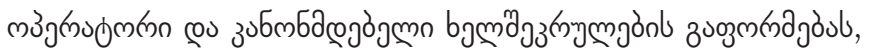

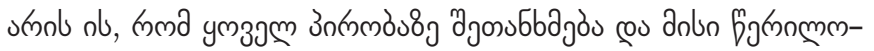

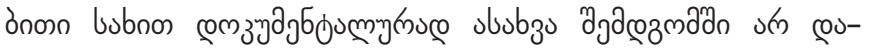

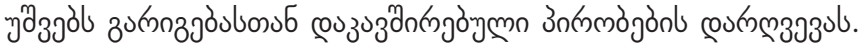

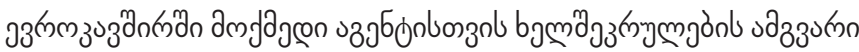

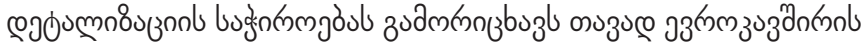

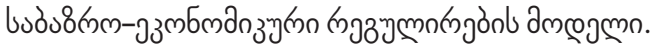

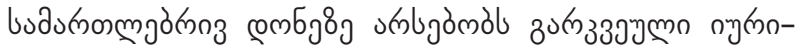

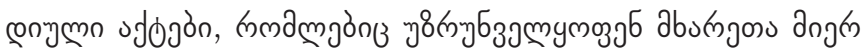

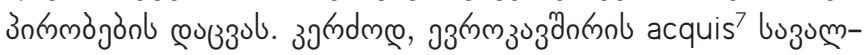

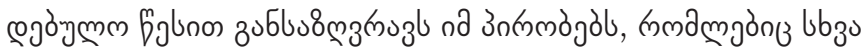

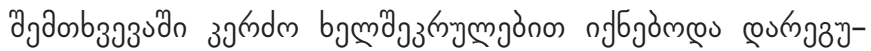

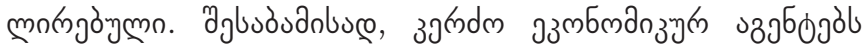

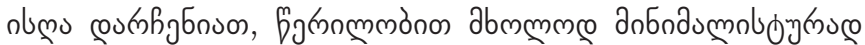

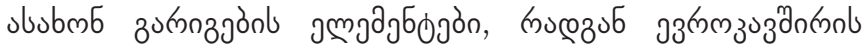

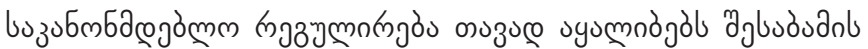

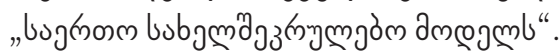

\section{cos335s}

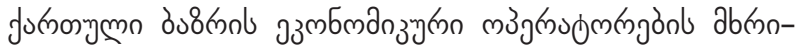

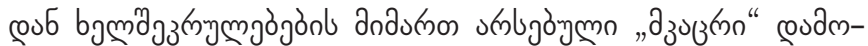

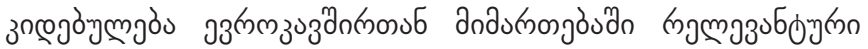
um sknol. bu8

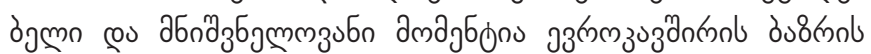

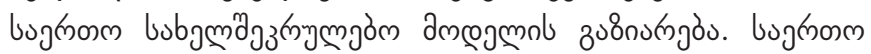

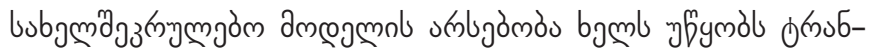

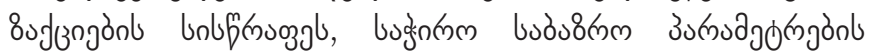

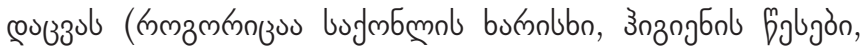

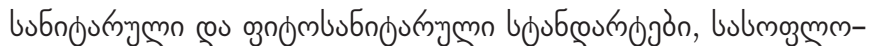

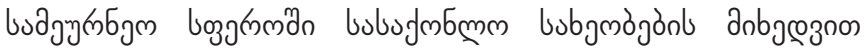

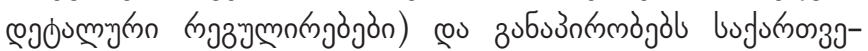

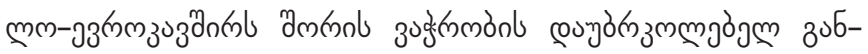

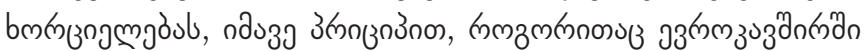
u ऊl

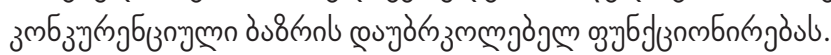

\footnotetext{
7 эк

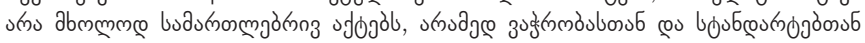

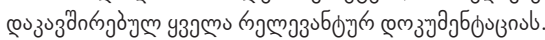

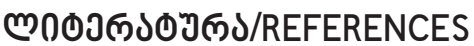

Bhala, R. (1997). Hegelian Reflections on Unilateral Action in the World Trading. 15 Berkeley Journal of International Economic Law 159, pp. 159-244.

Houh, E. M. (2003). Critical Interventions: Toward an Expansive Equality Approach to the Doctrine of Good Faith in Contract Law'. Cornell Law Review, Vol. 88, pp. 1025-1098.

Niamh Moloney. (2005 ). Building a Retail Investment Culture through Law: The 2004 Markets in Financial Instruments Directive. European Business Organization Law Review, Vol. 6, No. 3, pp. 341-422.

Zeitler, H. E. (September 2005). 'Good Faith' in the WTO Jurisprudence - Necessary Balancing Element or an Open Door to Judicial Activism?. Journal of International Economic Law, Volume 8, Issue 3, Pages 721-758.

The Impact of the Association Agreement and the Deep and Comprehensive Free Trade Agreement on Georgian consumers (October 6, 2019).http://asocireba.ge/files/file_2864583.

Access to the Official Journal. https://eur- lex.europa.eu/oj/direct-access.html

Consolidated Version of the Treaty on the Functioning of the European Union. https://eur-lex.europa.eu/resource. html?uri=cellar:2bf140bf-a3f8-4ab2-b506-fd71826e6da6.0023.02/DOC_2\&format=PDF.

The Legislative Herald of Georgia. https://matsne.gov.ge/ 